\title{
Comunicación organizacional en clave sonora: el caso de Blink, los podcasts de BBVA
}

\section{Organizational communication in sound format: The case of Blink, the BBVA's podcasts}

\author{
María-Luz Barbeito-Veloso; Juan-José Perona-Páez
}

Cómo citar este artículo:

Barbeito-Veloso, María-Luz; Perona-Páez, Juan-José (2019). “Comunicación organizacional en clave sonora: el caso de Blink, los podcasts de BBVA". El profesional de la información, v. 28, n. 5, e280511.

https://doi.org/10.3145/epi.2019.sep.11

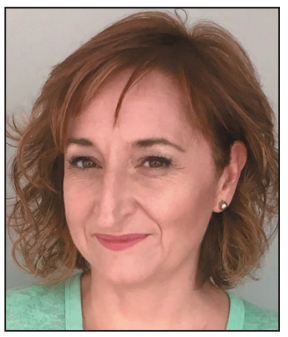
María-Luz Barbeito-Veloso $\square$
https://orcid.org/0000-0002-3159-7423
Universitat Autònoma de Barcelona
Facultat de Ciències de la Comunicació
Departament de Publicitat, Relacions Públiques i
Comunicació Audiovisual
Carrer de la Vinya, s/n. Edifici I,
08193 Bellaterra (Barcelona), España
mariluz.barbeito@uab.cat
Artículo recibido el 03-03-2019

Aceptación definitiva: 06-05-2019

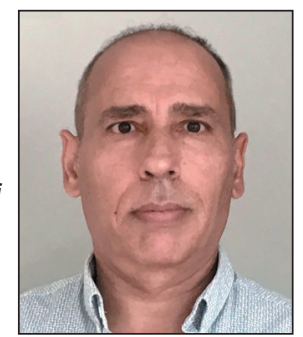

Juan-José Perona-Páez https://orcid.org/0000-0001-8256-9070

Universitat Autònoma de Barcelona Facultat de Ciències de la Comunicació Departament de Publicitat, Relacions Públiques i Comunicació Audiovisual Carrer de la Vinya, s/n. Edifici I, 08193 Bellaterra (Barcelona), España juanjose.perona@uab.cat

\section{Resumen}

La inclusión de podcasts en la estrategia comunicativa de las organizaciones es un fenómeno emergente, pues las empresas y las instituciones que confían en el audio branding para conectar con sus públicos saben que el sonido es una herramienta eficaz para transmitir sus activos inmateriales. Este artículo estudia el caso de Blink, los podcasts de BBVA, con el objetivo de evaluar la coherencia entre los contenidos que se difunden mediante este formato sonoro y los valores que proclama la entidad financiera. El análisis de contenido aplicado a la muestra, formada por todas las entregas de Blink emitidas durante su primer año de existencia (2018), evidencia una marcada correspondencia entre los mensajes y las prioridades estratégicas de la organización. El podcast se convertiría así en un instrumento que aporta un plus a la gestión estratégico-comunicativa de las corporaciones y refuerza el valor emocional de sus intangibles.

\section{Palabras clave}

Podcast; Comunicación organizacional; Comunicación corporativa; Organizaciones; Audio; Marcas; Contenido de marca; Audio branding; Valores; Estrategia; BBVA; Blink.

\begin{abstract}
The inclusion of podcasts in the communication strategy of organizations is an emerging phenomenon. There are many companies that rely on audio branding to connect with their audiences, and they know how sound could be an effective tool to communicate their intangible values. This article studies the case of Blink, the podcasts of BBVA. The goal is to evaluate the coherence between the content of corporate podcast and the values announced by the financial institution. It has been applied a content analysis to a sample consisting in whole of the Blink broadcast along its first year (2018). The results show a marked correlation between messages content and strategic priorities of the organization. Thus, podcasting could be a capable tool to add a plus to strategic-communicative management of corporations and to reinforce the emotional value of their intangibles.
\end{abstract}

\section{Keywords}

Podcasts; Podcasting; Organizational communication; Organizations; Audio; Branding; Branded content; Audio branding; Values; Corporate; Strategy; BBVA; Blink. 


\section{Introducción}

Las organizaciones llevan a cabo continuamente estrategias para mejorar los canales de comunicación con sus públicos, incorporando medios que aumenten la efectividad de sus acciones entre las marcas y sus stakeholders. Los mensajes sonoros siempre han formado parte de esos flujos comunicativos y la publicidad radiofónica ha sido una clara evidencia de la estrategia tradicional de empresas e instituciones para conectar con sus audiencias a través del oído. Como advierte Piñeiro-Otero (2015), los jingles, una de las manifestaciones que conforman la huella sonora de la marca, se erigieron en el formato publicitario estrella en los años 30-40 del siglo pasado. No obstante, y a pesar de la citada apuesta, un buen número de corporaciones han ido relegando a la radio a un segundo plano de sus actividades comunicativas porque consideran que el medio no permite "mostrar en imágenes" sus productos o servicios. Esta circunstancia ha derivado en campañas complementarias de bajo presupuesto, que han obedecido mucho más a la lógica del "efecto estar", es decir, a tener presencia en todas las plataformas convencionales, que no al efecto de retorno que se pudiera obtener (Balsebre et al., 2006). A ello se une que desde hace décadas la publicidad radiofónica padece un estancamiento creativo, que se ha visto reflejado tanto en los formatos y en la construcción de los mensajes, como en el uso del lenguaje sonoro y sus posibilidades informativas y expresivas (Perona-Páez, 2007; Espinosa-Mirabet; Vico-Blanco, 2016).

Sin embargo, el actual entorno digital está proporcionando nuevas oportunidades para el sonido y la explotación de su potencial comunicativo. Si la incorporación del transistor transformó los hábitos de escucha de los radioyentes, permitiendo poder seguir sus programas favoritos desde otro lugar que no fuera su casa, los actuales avances tecnológicos han roto las barreras que en cuanto a consumo y recepción sonora imperaban en la era analógica. Se dibuja así un escenario que, entre otras cosas, no sólo facilita nuevas vías de interacción con la audiencia, sino que amplía las posibilidades que la utilización del sonido puede tener para las organizaciones:

"si damos un paso más y nos referimos al audiobranding como un empleo estratégico del sonido, que se inserta en una concepción global e integrada de la marca, debemos señalar lo novedoso del fenómeno" (Piñeiro-Otero, 2015, p. 14-15).

Si el streaming permite una escucha a través de dispositivos conectados a internet, el podcast es un claro ejemplo de cómo los receptores ya no se limitan a sintonizar en cualquier momento y desde cualquier lugar con los temas que más le interesan mediante un sistema de descargas. De hecho, desde que este formato irrumpiera en 2004, son muchos los que han traspasado la frontera del "sólo escucho" y se han transformado en podcasters (creadores de podcasts), lo que ha contribuido al crecimiento de una oferta sonora cada vez más diversificada y altamente especializada. La facilidad en la gestión de la producción, que no requiere de grandes inversiones en infraestructuras para la elaboración y la difusión de los contenidos, así como la existencia de plataformas como iVoox, Soundcloud e iTunes, han facilitado el impulso de este fenómeno.

El alto grado de especialización de los podcasts es una de sus ventajas para alcanzar a los segmentos de la población que no ven cubiertas sus necesidades con la programación que ofrece la radio convencional. Esta circunstancia, además de incentivar la multiplicación de podcasters, ha llevado a las grandes cadenas de nuestro país a producir contenidos nativos online para sectores muy concretos (Bonet; Sellas, 2019). A pesar de que el consumo en España es aún muy inferior a las cuotas que alcanza en EUA, la curva de audiencia sigue una tendencia al alza:

"el consumo de podcasts se consolida y sigue aumentando cada año; ya en 2018 el 79\% de los oyentes de audio online escucha podcasts o emisoras puramente online en diferido/descarga" (IAB Spain, 2018, p. 3).

Las organizaciones no son ajenas a este fenómeno en crecimiento y, en su búsqueda constante de sistemas que propicien el diálogo y la interacción con sus stakeholders, han reparado en los beneficios que este tipo de productos pueden proporcionar en la construcción de sus marcas. Como advierte Geets:

"Ios podcasts son una excelente forma de acceder a una nueva audiencia y de hacerles conocer una marca. La inversión suele ser mucho menor que la del vídeo y tiene la ventaja añadida de expandirse a un formato relativamente infravalorado, pero de rápido crecimiento: la marca de audio. El podcast cada vez se está posicionando como una de las mejores opciones para llegar al público objetivo" (Geets, 2019).

Empresas como General Electric, UBS, Nintendo, Amazon, Spotify, Endesa, Iberia o Tinder han desarrollado contenidos específicos en formato podcast atendiendo a sus múltiples ventajas:

- la tematización a la que se hacía referencia, que permite llegar a nichos de audiencia muy concretos;

- el grado de intimidad que proporciona el sonido en relación con el oyente en el contexto de la sono-esfera digital (Perona-Páez; Barbeito-Veloso; Fajula-Payet, 2014; Barbeito-Veloso; Fajula-Payet; Enrique-Jiménez, 2012; Bull, 2010; Schafer, 1993); 
- la compatibilidad de la recepción sonora con la realización de otras actividades para unos oyentes que se caracterizan por su tendencia a la multitarea y la escucha positiva, puesto que son ellos mismos quienes deciden el momento del consumo y el dispositivo que van a utilizar.

El podcast también ayuda a crear comunidad entre los públicos, ya que el eje de los intereses comunes favorece la fidelización a partir del servicio de suscripción. A estos puntos fuertes, que van a contribuir a que la audiencia tenga una predisposición más amigable a recibir los mensajes, Rebold, empresa de marketing y comunicación basada en el análisis de datos, suma el bajo precio de las tarifas, la posibilidad de reutilizar contenidos producidos para otros formatos online y la escasa saturación de este mercado, lo que propicia un alto grado de diferenciación. También destaca la posibilidad de llegar a nuevas audiencias y aumentar el engagement con éstas a través del mayor recuerdo, el vínculo emocional que facilita el sonido y la posibilidad de crear plataformas de contenido de audio propio (Rebold, 2018).

Las razones expuestas explicarían que con independencia de las eventuales cuñas, menciones, patrocinios, etc., contratados en radio, cada vez sean más las empresas e instituciones que empiezan a ser conscientes de la importancia de incorporar el sonido tanto en el conjunto de sus estrategias de comunicación como en la construcción de su identidad corporativa, lo que se está traduciendo en una clara expansión del audio branding (Vives, 2014; Gustafsson, 2015) y de sus principales manifestaciones. El audio branding irrumpe con fuerza cuando las corporaciones comprueban el retorno y por eso,

"las marcas deben tener una identidad de audio única, reconocible y relevante que se transmita a través de todas sus comunicaciones. En la era de los altavoces inteligentes, esto podría significar que las marcas desarrollen su propia voz hablada" (IAB Spain, 2018).

Siguiendo a Bronner, Allan (2015) sostiene que los elementos comunes que conforman el audio branding incluyen el audio-logo, los jingles, la canción de marca, las voces corporativas, el icono sonoro, los paisajes de marca y el tema corporativo. A esta clasificación podrían añadirse

"los eventos musicales, las plataformas 2.0 y las radios corporativas, que se acercarían más al contenido de marca" (Piñeiro-Otero, 2015, p. 678).

En el caso de las emisoras corporativas, la organización establece los criterios programáticos que se ajusten a los parámetros de la identidad que se quiera trasmitir a un público concreto. Por tanto, se podría afirmar que en sí misma la emisora se convierte en el propio mensaje.

"Radio corporativa es aquella que parte de un estudio de marketing. Se estudia la marca, cuáles son sus objetivos, el público objetivo, en qué nivel se sitúa (alto - medio - bajo) y según los resultados se crea un canal, una radio, exclusiva para ese cliente. Siempre amparado por una plataforma en internet y con un player en cada uno de los establecimientos con una IP address única para poder acceder de manera individualizada" (Peinado-Miguel; Rodríguez-Barba; Rodríguez-Pallarés, 2017, p. 151).

\section{Objeto de estudio, método y preguntas de investigación}

Esta investigación se centra en el podcast como apuesta estratégica de contenido de marca, lo que se denomina brand podcasting, branded podcast o podcast corporativo y, por tanto, como un elemento más de audio branding. Es decir, no se va a estudiar el podcast como soporte de anuncios y/o patrocinios, sino como relato de marca que se elabora de forma específica con objetivos organizacionales concretos. Su pretensión, a partir del estudio de caso, es averiguar hasta qué punto el podcast se revela para una determinada empresa o institución como un instrumento de comunicación externa, si su línea editorial es coherente con su estrategia corporativa y si, en consecuencia, su uso ayuda a reforzar la imagen intencional de la organización. El estudio de caso permite investigar un fenómeno contemporáneo en su contexto real, aunque Yin advierte que los límites entre el fenómeno y el contexto no son claramente evidentes (Yin, 2003).

Para la consecución de este objetivo, el análisis se aplicará sobre el producto Blink, los podcasts de BBVA, que inició su andadura el 22 de diciembre de 2017. Si se ha seleccionado la compañía BBVA es porque tradicionalmente ha mostrado un notable interés por transmitir con sonidos algunos de sus principales intangibles, lo que la ha convertido en un referente en la implementación del audio branding en sus planes de comunicación. Blink, elaborado por el equipo de BBVA y conducido por los periodistas Lartaun de Azumendi y Reyes Pariente, es una propuesta sonora de periodicidad semanal, disponible en iVoox, Apple Podcast, bbva.com y las redes sociales corporativas de la organización, que ofrece

"información práctica sobre finanzas personales, soluciones fintech, metodologías de trabajo y consejos para que los usuarios tomen las mejores decisiones financieras" (BBVA, 2018).

Se trata de una iniciativa que consolida la renovación de la identidad sonora que el banco llevó a cabo en 2017 . El cambio del eslogan Adelante por el lema Creando oportunidades ha traído un nuevo sonido para la marca. Desarrollada por AmpSound Branding, la nueva melodía de BBVA pretende "aportar dinamismo, optimismo, autenticidad, transparencia..." (MarketingNews, 2019). Con Blink se quiere ofrecer a los usuarios información que les permita tomar las mejores decisiones financieras de una forma fácil y accesible.

En palabras de Nacho Jiménez Soler, director de estrategia de comunicación del Grupo BBVA, esta iniciativa 
“es un primer paso en el desarrollo de contenidos por voz que complementará la estrategia de desarrollo de la voz propia del grupo (BBVA, 2018).

Lo que se busca a través de contenidos de utilidad, accesibles y fácilmente compartibles, es implementar estrategias para ganar en cuota de atención. Después de Blink han llegado otras propuestas programáticas audiovisuales como Aprendemos juntos, Data historias y Deep talks, las cuales no han sido consideradas en este trabajo por distanciarse del formato podcast exclusivamente sonoro.

La muestra está compuesta por 49 podcasts de Blink emitidos entre diciembre de 2017 y diciembre de 2018. Si bien a lo largo del año observado se publicaron 50 episodios, el último -que es un resumen de los anteriores- se ha desestimado para neutralizar posibles distorsiones. Dicha muestra supone algo más de 16 horas y 23 minutos de podcast. El diseño metodológico se ha basado en un análisis que ha permitido extraer información sobre seis aspectos:

- identificación del podcast (número de entrega, día de publicación, título);

- contenidos por secciones (tema del día, curiosidades, otros temas);

- alusiones directas a la marca;

- acciones asociadas a la marca;

- adjetivos asociados a la marca;

- actores.

Cuando hablamos de actores nos referimos a aquellas personas a las que se les da voz en el podcast, en calidad de responsables de la entidad financiera, expertos en algún tema concreto o usuarios del banco:

"Ias estrategias publicitarias que se utilizan para que la audiencia perciba un elevado grado de interés o implicación del prescriptor con el producto, servicio o marca anunciado son, por un lado, utilizar el estilo testimonial, en el que el portavoz se declara usuario del producto y, por otro lado, utilizar la primera persona en la locución del mensaje, lo que da cabida a distintos argumentos de venta" (Perelló-Oliver; Muela-Molina; Campos-Zabala, 2018, p. 1167).

Además de los aspectos señalados, también se han considerado otras cuestiones formales:

- duración de cada episodio;

- número de locutores/as que intervienen en cada edición;

- músicas utilizadas;

- efectos sonoros;

- géneros programáticos.

En España, un precedente directo relacionado con lo que plantea esta investigación se encuentra en el trabajo de Sellas (2018) sobre el podcasting como medio de identidad corporativa. Sellas evalúa los nueve capítulos del podcast Aerolínea momentos, coproducido por Podium Podcast e Iberia con motivo del 90 aniversario de la aerolínea y, aunque advierte que el uso del podcasting en las tácticas corporativas es todavía incipiente, su estudio revela un buen anclaje del mismo en la estrategia de la organización analizada. En nuestro caso, y en sintonía con los objetivos generales anteriormente expuestos, los datos extraídos permitirán dar respuesta a preguntas de investigación como las siguientes:

¿Los contenidos que se difunden a través del podcast son coherentes con la estrategia corporativa de la organización? ¿Hasta qué punto existe una correlación entre los mensajes sonoros y los valores de la empresa?

\section{Resultados}

En 2012 BBVA se definía (misión) como un grupo global de servicios financieros cuyo principal compromiso consistía en aportar las mejores soluciones a sus clientes, un crecimiento rentable y sostenido a sus accionistas, y progreso en las sociedades en las que estaba presente. Paralelamente su visión se articulaba en torno al eje "trabajamos por un futuro mejor para las personas".

A partir de 2017, y tras un proceso de transformación de su identidad corporativa, la entidad financiera fija su aspiración en afianzar la relación con sus clientes, y su propósito en

"poner al alcance de todos las oportunidades de esta nueva era a través de productos y servicios que ayuden a las personas a tomar mejores decisiones financieras y a cumplir sus objetivos vitales" (BBVA, 2017).

La triangulación de sus tres principios -integridad, prudencia y transparencia- desembocaba entre 2006 y 2017 en siete valores corporativos:

1) cliente

2) creación de valor

3) equipo

4) estilo de gestión

5) ética e integridad

6) innovación

7) responsabilidad social corporativa. 
Desde 2017 (tabla 1), esos valores se han transformado en tres (BBVA, 2017):
1) el cliente es lo primero
2) pensamos en grande
3) somos un solo equipo,

al tiempo que se han implementado seis prioridades estratégicas:

1) la mejor experiencia de cliente

2) ventas digitales

3) nuevos modelos de negocio

4) optimización de la asignación de capital

5) liderazgo en eficiencia

6) el mejor equipo.

Tabla 1. Principios de identidad corporativa de la organización investigada

\begin{tabular}{|c|c|c|c|}
\hline Lema & "Adelante" (2006-2017) & Lema & "Creando oportunidades" (a partir de 2017) \\
\hline Misión & $\begin{array}{l}\text { Un grupo global de servicios financieros cuyo prin- } \\
\text { cipal compromiso es aportar las mejores soluciones } \\
\text { a sus clientes, un crecimiento rentable y sostenido a } \\
\text { sus accionistas y progreso en las sociedades en las } \\
\text { que está presente. }\end{array}$ & Propósito & $\begin{array}{l}\text { Poner al alcance de todos las oportunidades de } \\
\text { esta nueva era a través de productos y servicios que } \\
\text { ayudan a las personas a tomar mejores decisiones } \\
\text { financieras y cumplir sus objetivos vitales. }\end{array}$ \\
\hline Visión & Trabajamos por un futuro mejor para las personas. & Aspiración & Afianzar la relación con sus clientes. \\
\hline Principios & $\begin{array}{l}\text { 1. Integridad } \\
\text { 2. Prudencia } \\
\text { 3. Transparencia }\end{array}$ & $\begin{array}{l}\text { Prioridades } \\
\text { estratégicas }\end{array}$ & $\begin{array}{l}\text { 1. La mejor experiencia de cliente } \\
\text { 2. Ventas digitales } \\
\text { 3. Nuevos modelos de negocio } \\
\text { 4. Optimización de la asignación de capital } \\
\text { 5. Liderazgo en eficiencia } \\
\text { 6. El mejor equipo }\end{array}$ \\
\hline Valores & $\begin{array}{l}\text { 1. Cliente } \\
\text { 2. Creación de valor } \\
\text { 3. Equipo } \\
\text { 4. Estilo de gestión } \\
\text { 5. Ética e integridad } \\
\text { 6. Innovación } \\
\text { 7. Responsabilidad social corporativa }\end{array}$ & Valores & $\begin{array}{l}\text { 1. El cliente es lo primero } \\
\text { 2. Pensamos en grande } \\
\text { 3. Somos un solo equipo }\end{array}$ \\
\hline
\end{tabular}

Fuente: Elaborado a partir de la información de bbva.com

A continuación se exponen los datos más relevantes arrojados por el análisis de contenido, los cuales, como ya se avanzaba, van a permitir dilucidar si existen puntos de conexión entre los valores y las líneas estratégicas definidos por la organización en 2017. Por otra parte, dichos datos servirán igualmente para comprobar la coherencia comunicativa del podcast y su contribución a la construcción de identidad de marca de la empresa, todo ello con el objetivo de dar respuesta a las preguntas de investigación anteriormente formuladas.

En las 49 entregas de Blink estudiadas se desarrollan 110 asuntos distribuidos en tres secciones: tema del día (49), que en 23 ocasiones es un monográfico que ocupa todo el podcast, tema secundario (26) y curiosidades (35). La mayoría de los aspectos tratados se refieren a educación financiera y personas (38\%), aunque seguidos muy de cerca por materias relacionadas con la nueva era (29\%), tales como digitalización, big data, ciberseguridad, coches autónomos, buscadores en internet, apps, etc. El resto se centra en cuestiones relativas a oportunidades (12\%) -sobre todo de emprendimientoproductos y servicios (10\%), decisiones financieras (8\%) y objetivos vitales (3\%). Es destacable que en ocho de las once veces en las que se abordan productos y servicios se explican los de la propia organización (aplicaciones tipo Tuyyo, Servicio de alta inmediata, Android pay, Bizum, entre otras), dando un especial protagonismo al contenido de marca o branded content.

En la muestra investigada se contabilizan 703 alusiones directas a BBVA, lo que supone una media de 14,3 menciones por podcast. Se trata de una cifra notable, ya que teniendo en cuenta que la duración media de las entregas se sitúa en torno a los 20 minutos, la organización se nombra de media 0,7 veces por minuto. A la luz de estos datos, el podcasting corporativo analizado se revela como un canal comunicativo de importante impacto publicitario debido a la reiteración de la marca -la cual se integra periódica y armónicamente a lo largo del discurso sonoro- y la consiguiente estimulación del recuerdo. La alusión continuada a la entidad financiera en el transcurso de los podcasts guarda una estrecha relación con el asunto desarrollado, como demuestra el hecho de que las dos entregas con mayor número de menciones (29), publicadas el 12 de marzo y el 25 de mayo de 2018, versan sobre BBVA Open Talent (la competición fintech de referencia) y Banca móvil global (la app de BBVA) respectivamente. Por el contrario, los dos podcasts con el menor número de referencias expresas a la marca (7)

El podcast corporativo contribuye a la transmisión de los valores intangibles de las empresas e instituciones 
tratan sobre coches autónomos (5/11/2018) y altavoces inteligentes (17/12/2018), dos temas que, aun siendo de actualidad, poco tienen que ver con el perfil de la empresa estudiada. A partir de la emisión número 15, del 12 de marzo de 2018, el resumen escrito que aparece junto a cada podcast incorpora enlaces con páginas de la organización en las que se amplía información sobre los asuntos tratados, o bien con el perfil que los personajes a los que se da voz tienen en LinkedIn. Esta práctica hipertextual redunda en la construcción de identidad y favorece la visibilidad tanto de las acciones que lleva a cabo el banco como de las personas que en él trabajan.

El análisis de contenido permite observar la citación directa de 57 acciones atribuidas a la organización (por ejemplo: "crea planes a medida para mejorar la salud financiera de las personas", "difunde conocimientos", "mejora la vida de sus empleados", "contribuye a la creación de energía renovable", etc.) Estas acciones -señaladas explícitamente por los conductores del podcast o por los personajes a los que se da voz-contribuyen a trazar el perfil de una marca que:

- facilita: desde la relación con las grandes empresas hasta la integración social;

- ayuda: a las empresas, a los clientes, a edificar una sociedad más responsable...;

- busca: la mejor experiencia de cliente, el mayor impacto positivo, sorprender al cliente, agradar al cliente...;

- impulsa: el desarrollo sostenible, la movilidad interna, los negocios que ya están en marcha...;

- crea: experiencias de cliente, planes a medida para mejorar la salud financiera, productos nuevos...

Las acciones mencionadas entroncan con cuatro de las prioridades estratégicas fijadas por BBVA en 2017, siendo "el mejor equipo" (en 22 ocasiones) y "la mejor experiencia de cliente" (en 18) las que más sobresalen en las entregas de Blink (gráfico 1). Por el contrario, "las ventas digitales" y "la optimización de la asignación de capital" no están representadas por ninguna de las acciones señaladas, mientras que "liderazgo en eficiencia" (12) y "nuevos modelos de negocio" (5) ocupan posiciones más modestas.

Los adjetivos asociados a la organización se erigen también en un indicador mediante el que es posible testar la coherencia comunicativa del formato sonoro con la identidad empresarial. Se han detectado 25 calificaciones directas, las cuales dibujan una organización que es ante todo:

- pionera: en la lucha contra el cambio climático, en la apuesta por el fintech, en el uso de la realidad aumentada en el campo de la vivienda...;

- líder: en banca móvil, en bonos verdes...;

- innovadora;

- comprometida: con las personas, con la sociedad...

El $56 \%$ de los adjetivos encontrados se identifican con el valor "pensamos en grande"; el $24 \%$ con "somos un solo equipo"; y el $20 \%$ con "el cliente es lo primero". La organización muestra una especial sensibilidad por comunicar sus principales intangibles, como prueba el hecho de que el 27 de julio de 2018 Blink, con el título ¿Por qué es importante para una empresa tener valores? estuviera íntegramente dedicado al día de los valores de BBVA.

El podcasting investigado es un producto informativo en el que la voz impera claramente sobre el resto de componentes del lenguaje sonoro. Si bien la música aparece en todas las entregas, su protagonismo es ínfimo, en tanto que generalmente se mantiene en un discreto tercer plano y, cuando sube a primero - una media de 7 veces por emisión- no pasa de ser un simple separador entre bloques (función sintáctico-gramatical). Las únicas excepciones a esta regla se observan en la sección "curiosidades" dedicada al carnaval en América Latina (12/02/2018) y la gastronomía en Semana Santa en España y América Latina (19/03/2018), en las que sí se incluyeron músicas en función descriptivo-ubicativa, es decir melodías no diegéticas que trasladan mentalmente al oyente a un lugar o a una época concretos.

Los efectos sonoros tienen una presencia todavía mucho más limitada, hasta el punto de que únicamente se detecta su inclusión en tres ocasiones: cuando se habla de las preferencias de pago en efectivo o con tarjeta, de los coches autónomos y de los altavoces inteligentes. Esta circunstancia Ilama especialmente la atención desde la perspectiva de la
Los podcasts de BBVA muestran una marcada correlación entre los contenidos que se difunden y las prioridades estratégicas de la organización 
creación sonora, puesto que pone de manifiesto la infrautilización de un recurso que en muchos casos sólo aparece como simple apoyo al lenguaje verbal, sin aporte de información semántica adicional.

En géneros, la entrevista informativo-interpretativa y las declaraciones de expertos destacan como soporte para el desarrollo de los contenidos. La citada modalidad de entrevista está presente en un $55 \%$ de los podcasts. El predominio de los géneros informativos señalados comporta que a lo largo de 2018 Blink dé voz a 120 personas -83 hombres $(69,1 \%)$ y 37 mujeres $(30,9 \%)$ - la mayoría $(60,8 \%)$ integrantes de la propia organización. Ocho de esas personas, en este caso hombres todos ellos vinculados al banco, aparecen más de una vez, especialmente el presidente de la empresa, que lo hace en 7 ocasiones, y el consejero delegado, en 4 (tabla 2). La entrevista se utiliza para dar voz a 37 de los 120 sujetos que intervienen en el podcast, 25 hombres $(67,5 \%)$ y 12 mujeres $(32,5 \%)$, de los cuales $33(89,2 \%)$ pertenecen a la entidad financiera. El responsable de divulgación analítica y el responsable de análisis macroeconómico son entrevistados en dos ocasiones, mientras que dos de las cuatro personas entrevistadas ajenas a la empresa están vinculadas a organizaciones sin ánimo de lucro ( $m Y m O$ e itwillbe.org), una a Nexe y la otra a Amazon.

La notable presencia de voces de la entidad convierte al producto sonoro en un formidable instrumento de comunicación externa susceptible de utilizarse para que los miembros de una determinada organización se erijan en portavoces de su cultura corporativa y como elemento de identificación con los destinatarios del mensaje:

“a través de los empleados de una compañía se consigue empatizar y conectar con el público. Lo que se busca es poner en valor a las personas que hay detrás de un determinado producto o servicio, y mediante eso diferenciarse con una historia auténtica y real" (Foromarketing, 2018).

No obstante, su uso también puede propiciar que afloren, como muestra este estudio de caso, estructuras organizacionales netamente dominadas por hombres, con las connotaciones negativas que este hecho puede comportar para su reputación e imagen social.

\section{Conclusiones}

La transmisión de los activos intangibles, a los que cada vez se les confiere una mayor importancia estratégica, se perfila como uno de los retos que tienen las empresas y las instituciones a la hora de explotar tanto las posibilidades que brinda el branding holístico como la multiplicación, gracias a los avances tecnológicos, de los puntos de contacto con sus públicos. Ante este reto, entre las organizaciones proliferan las acciones de comunicación que sobrepasan las fronteras de lo estrictamente racional y buscan despertar sensaciones y emociones en sus consumidores y usuarios, pero sobre todo generar experiencias sensitivas que favorezcan una relación positiva con las mismas.

Aunque el oído ocupa todavía un lugar poco destacado como herramienta de estimulación, cada vez son más las corporaciones que sitúan al sonido como un elemento a considerar en la definición de su identidad corporativa. En los últimos años, por ejemplo, la práctica totalidad de las compañías automovilísticas se han dotado de audio-logo, al tiempo que ha aumentado el número de las que, fuera de este sector, también disponen de él. Sin ir más lejos, en febrero de 2019 Mastercard estrenó un logo sonoro que se puede oír, como explica García (2019), en momentos muy concretos del costumer journey, como después de haber pagado con la tarjeta, en los tonos de espera de atención al cliente, al finalizar los vídeos publicitarios, etc. Por otro lado, también han crecido tanto los establecimientos que presentan una cuidada ambientación sonora como el número de organizaciones que aprovechan los nuevos soportes, entre ellos el podcast, para crear contenido de marca.

Según los resultados de esta investigación, el podcast se manifiesta como un instrumento que aporta un plus a la gestión estratégico-comunicativa de las entidades en un escenario multiplataforma, a la vez que refuerza el valor de sus activos intangibles. Y todo ello en un entorno tecnológico que favorece el aumento de mensajes sonoros de mayor accesibilidad y susceptibles de ser incorporados en distintos contextos para llegar a todos los puntos de contacto con los públicos.

El estudio de caso evidencia que el podcast corporativo muestra una correlación muy destacada entre los contenidos que se trasmiten y los valores de la empresa que lo explota, por lo que se consagra como un formato para, además de su capacidad de generar branded content, divulgar sus acciones de responsabilidad social corporativa (RSC). La entidad financiera estudiada no escatima esfuerzos en subrayar, en el momento de explicitar sus
En el caso estudiado, el podcast se revetenido de marca y divulgar acciones de responsabilidad social corporativa la como un formato para generar con- 
actividades, su compromiso con el desarrollo sostenible, el consumo responsable, la lucha contra el cambio climático o la integración social, por ejemplo.

Por otro lado, y pese a las debilidades detectadas en cuanto a riqueza sonora, el producto examinado presenta una marcada coherencia con las prioridades de la organización, por lo que los resultados confirmarían y consolidarían el buen anclaje entre las estrategias organizacionales y este formato, algo que ya constataba Sellas (2018) en su estudio sobre el podcast de Iberia con motivo de su 90 aniversario.

No obstante, a medida que se implementen sistemas de medición que ayuden a entender el comportamiento de la audiencia en relación con los podcasts será más fácil analizar la efectividad de su inclusión en los planes estratégicos empresariales e institucionales, y, especialmente, su utilidad en la gestión de la imagen y reputación organizacional.

\section{Referencias}

Allan, David (2015). "Audiobranding and ROI. A review". In: Bronner, Kay; Cornelius, Ringe; Hirt, Rainer. Audio branding yearbook 2014/2015. Baden-Baden: Nomos Edition, pp. 103-116. ISBN: 9783848717330

Balsebre, Armand; Ricarte, José-María; Perona-Páez, Juan-José; Roca, David; Barbeito-Veloso, Mariluz; Fajula-Payet, Anna (2006). Los mitos de la publicidad radiofónica. Estrategias de la comunicación publicitaria en la radio española. Madrid: Cátedra. ISBN: 8437622913

Barbeito-Veloso, Mariluz; Fajula-Payet, Anna; Enrique-Jiménez, Ana-María (2012). “Creativity: the key to creating successful advertising messages in the digital sonosphere". In: Oliveira, Madalena; Portela, Pedro; Santos, Luis-António (eds.). Radio evolution: Conference proceedings. Braga (Portugal): Universidade do Minho, pp. 295-303. ISBN: 978989 9724495

http://www.lasics.uminho.pt/ojs/index.php/radioevolution/article/view/853/802

BBVA (2018). "BBVA lanza un canal podcast para reforzar la difusión de sus contenidos propios". BBVA, 10 enero. https://www.bbva.com/es/bbva-lanza-canal-podcast-reforzar-difusion-contenidos-propios

BBVA (2017). "Estrategia y modelo de negocio". BBVA.

https://accionistaseinversores.bbva.com/microsites/cuentasanuales2017/informe-de-gestion/estrategia-y-modelo-denegocio/index.html

Bonet, Montse; Sellas, Toni (2019). "Del flujo al stock: el programador radiofónico ante la gestión del catálogo digital”. El profesional de la información, v. 28, n. 1, e280109.

https://doi.org//10.3145/epi.2019.ene.09

Bull, Michael (2010). "iPod: un mundo sonoro personalizado para sus consumidores”. Comunicar, v. 17, n. 34, pp. 55-63. https://doi.org/10.3916/C34-2010-02-05

Espinosa-Mirabet, Sílvia; Vico-Blanco, Maria (2016). “La involución de los formatos comerciales radiofónicos: España 10 años después. Zer. Revista de estudios de comunicación, v. 21, n. 41, pp. 227-243.

http://www.ehu.eus/ojs/index.php/Zer/article/view/17293/15090

Foromarketing (2018). "Los empleados como estrategia de marketing y publicidad". Foromarketing, 10 octubre. https://www.foromarketing.com/los-empleados-estrategia-marketing-publicidad

García, Modesto (2019). "Mastercard estrena un logo sonoro que escucharemos cada vez que paguemos". Brandemia. org, 18 febrero.

http://brandemia.org/mastercard-estrena-un-logo-sonoro-que-escucharemos-cada-vez-que-paguemos

Geets, Kevin (2019). “El éxito del 'podcast' y cómo tu marca puede aprovecharlo”. MarketingNews, 8 mayo. http://www.marketingnews.es/tecnologia/opinion/1132179054405/exito-del-podcast-y-marca-puede-aprovecharlo.1.html

Gustafsson, Carl (2015). "Sonic branding: A consumer-oriented literature review". Journal of brand management, v. 22, n. 1, pp. 20-37.

https://link.springer.com/article/10.1057/bm.2015.5

IAB Spain (2018). Top tendencias digitales 2019. Madrid: IAB Spain.

https://iabspain.es/estudio/top-tendencias-digitales-2019

MarketingNews (2019). "Esta es la identidad sonora de BBVA". MarketingNews, 21 febrero.

http://www.marketingnews.es/marcas/noticia/1105482054305/identidad-sonora-de-bbva.1.html

Peinado-Miguel, Fernando; Rodríguez-Barba, Dolores; Rodríguez-Pallarés, Miriam (2017). "Hacia un nuevo modelo de negocio radiofónico: la radio corporativa”. Estudios sobre el mensaje periodístico, v. 23, n. 1, pp. 147-164.

https://doi.org/10.5209/ESMP.55588 
Perelló-Oliver, Salvador; Muela-Molina, Clara; Campos-Zabala, María-Victoria (2018). “La personificación en la publicidad radiofónica. Análisis de la presencia y uso de la figura del portavoz". Revista latina de comunicación social, n. 73, pp. 1163-1178.

https://doi.org/10.4185/RLCS-2018-1301en

Perona-Páez, Juan-José (2007). “Formatos y estilos publicitarios en el prime-time radiofónico español: infrautilización y sequía de ideas". Zer. Revista de estudios de comunicación, v. 12, n. 23, pp. 219-242.

http://www.ehu.eus/ojs/index.php/Zer/article/view/3658/3290

Perona-Páez, Juan-José; Barbeito-Veloso, Mariluz; Fajula-Payet, Anna (2014). “Los jóvenes ante la sono-esfera digital: medios, dispositivos y hábitos de consumo sonoro". Comunicación y sociedad, v. 27, n. 1, pp. 205-224.

https://www.unav.edu/publicaciones/revistas/index.php/communication-and-society/article/view/36011

Piñeiro-Otero, Teresa (2015). "Del jingle a las radios corporativas: una aproximación al concepto de audiobranding". Prisma social. Revista de ciencias sociales, n. 14, pp. 663-668.

http://www.isdfundacion.org/publicaciones/revista/numeros/14/secciones/abierta/nbiblio_01_jingle_radios.html

Rebold (2018). "Rebold analiza el incremento de los ingresos publicitarios a través de podcasts". Rebold, 13 septiembre. https://letsrebold.com/es/blog/digilant-analiza-el-incremento-de-los-ingresos-publicitarios-a-traves-de-podcasts

Schafer, R. Murray (1993). The soundscape: Our sonic environment and the tuning of the world. Rochester, VT: Destiny books. ISBN: 0892814551

Sellas, Toni (2018). "El uso del podcasting como herramienta de la identidad corporativa en la campaña del 90 aniversario de Iberia. El caso del podcast Aerolínea Momentos". Communication papers: Media literacy and gender studies, v. 7, n. 15, pp. 155-170.

http://ojs.udg.edu/index.php/CommunicationPapers/article/view/430/pdf

Vives, Ramón (2013). “Audiobranding: el poder del sonido y la música aplicado al marketing”. PuroMarketing, 14 mayo. http://www.puromarketing.com/44/16154/audiobranding-poder-sonido-musica-aplicados-marketing.html

Yin, Robert K. (2003). Case study research. Design and methods. 3rd ed.. London: Sage Publications. ISBN: 076192552 X

\section{Inforảrea}

\section{Ayudamos a tu organización en la transformación digital y el gobierno de la información}

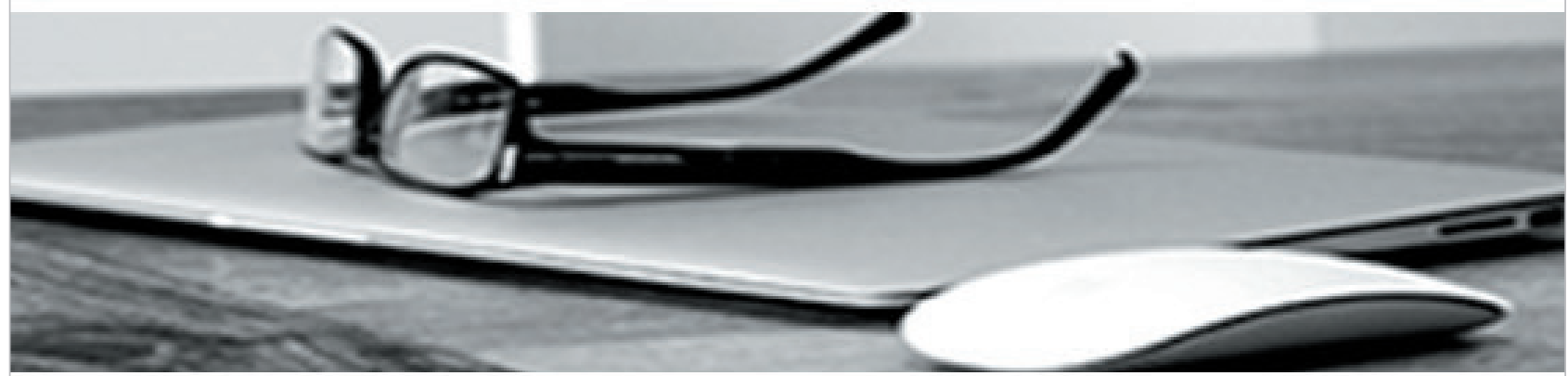

* Consultoría estratégica en gestión y gobierno de la información

* Gestión documental y "records management"

* Gestión de contenidos, intranets corporativas y entornos de colaboración

* Estudios especializados

Clientes satisfechos, cientos de empresas nacionales e internacionales y más de 30 años de experiencia son la mejor garantía de nuestra reputación. 